Fontan (2011)

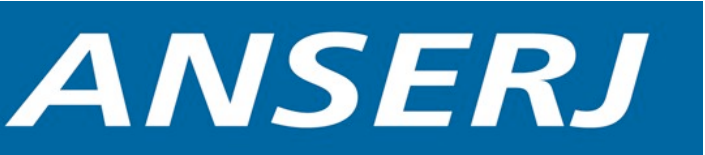

Vol. 2, No 2

Fall / Automne 2011

pp. $37-56$

Canadian Journal of Nonprofit and Social Economy Research

Revue canadienne de recherche sur les OBSL et I'économie sociale

\title{
Entreprenariat social et entreprenariat collectif : synthèse et constats
}

\author{
Jean-Marc Fontan
}

Université du Québec à Montréal (UQAM)

\section{RÉSUMÉ}

L'article présente les résultats d'une recherche portant sur les notions d'entrepreneur social et d'entrepreneur collectif. Après avoir défini le concept d'entrepreneur, nous voyons comment les qualitatifs «social » et «collectif» transforment l'idée d'entreprendre en un projet plus intégré où les objectifs sociaux, politiques et économiques sont «harmonieusement » agencés. La recherche repose essentiellement sur la réalisation d'une revue de littérature de textes clés produits en langue française et anglaise en Amérique du Nord et en Europe. L'analyse des données recueillies nous permet de présenter des critères simples pour proposer une définition de ces notions qui devrait rendre compte de la réalité des pratiques rencontrées sur le terrain et des contributions théoriques et critiques faites par les auteurs et travaux consultés. Elle permet aussi de présenter une dizaine de constats qui illustrent tant les points de convergence que les divergences observées entre les types d'entreprenariat étudiés.

\section{ABSTRACT}

This article presents the results of a study bearing on the notions of social entrepreneur and collective entrepreneur. After defining the concept of entrepreneur, I look at how the adjectives "social" and "collective" transform the project of being an entrepreneur into a more integrated one where the social, political and economic objectives are "harmoniously" organized. The study is essentially based on a literature review of key English- and French-language texts written in North America and in Europe. The analysis of the collected data allows me to present simple criteria for proposing a definition of these notions that should take into account both the reality of the practices encountered in the field and the theoretical and critical contributions made by the authors and the texts that I consulted. It also enables me to present ten observations that illustrate both the points of convergence and divergence between the types of entrepreneurship that I studied.

\section{Mots clés / Keywords}

Économie sociale; Entreprise; Entreprise collective; Entreprise sociale; Organisme à but non lucrative / Social economy; Enterprise; Collective enterprise; Social enterprise; Nonprofit organization 


\section{Fontan (2011)}

\section{INTRODUCTION}

L'objet de cet article est de présenter une réflexion sur l'entreprenariat social et l'entreprenariat collectif à partir d'une revue de la littérature effectuée à l'aide de documents nord-américains et européens. Pourquoi cette réflexion? Pour une raison relativement simple. II existe toujours une confusion de sens entre les types d'entreprenariat développés par des acteurs privés, publics, sociaux ou collectifs. De plus, tant dans le monde universitaire que dans celui des acteurs terrain, nous observons la présence d'un débat critique sur les différentes définitions, interprétations et vertus associées à « l'entreprenariat social » (Hodgson, 2004; Bull, 2008; Draperi, 2010).

La question de la responsabilité sociale des entreprises et le débat qu'elle provoque dans les milieux associatifs (Salmon, 2011) soulèvent des attentes claires. II est important de pouvoir comprendre les univers culturels propres aux différents types d'entreprenariat. II est tout aussi central de bien caractériser les points de convergence et de divergence qui existent entre la pratique entrepreneuriale privée ou publique et les pratiques entrepreneuriales sociales et collectives. Enfin, il importe de montrer la parenté historique commune entre ces différentes formes d'entreprenariat. Notre article fournit des éléments critiques de réponse permettant de tracer les différences entre ces réalités.

Pour réaliser cette revue de la littérature, nous avons travaillé à partir d'un corpus de textes écrits en langues française et anglaise. II s'agit principalement de textes nord-américains et européens. Ces textes ont été publiés par des chercheurs universitaires ou par des représentants d'organisations vouées à l'étude ou à la promotion de l'entreprenariat social.

Pour repérer les textes clés, nous avons identifié des textes canadiens qui présentent une synthèse sur la notion d'entreprenariat social telle qu'elle se présente au Canada1. Dans un deuxième temps, nous avons exploré la littérature états-unienne, plus riche, plus ancienne et plus diversifiée2. Enfin, nous nous sommes penché sur la littérature européenne principalement à partir de textes produits au Royaume-Uni, en Belgique, en Italie ou en France 3 .

La recherche documentaire a été effectuée principalement sur Internet et de façon moins importante à partir d'articles de revues ou de livres spécialisés. Les textes relèvent de travaux menés par des chercheurs universitaires, par des consultants au service d'organisations gouvernementales ou par des chercheurs/consultants œuvrant pour des fondations privées.

Parmi les textes que nous avons consultés, nombreux sont ceux qui ont été conçus pour servir de guide à l'action. Ils ont été produits dans une perspective de promotion et de développement de l'entreprenariat social. Notre revue tient certes compte de cette perspective, tout en ajoutant une recension d'écrits plus théoriques. Ces écrits posent un regard réflexif sur la pratique globale de l'entreprenariat social.

Enfin, en raison de son ampleur, cette revue de littérature complète des travaux récents importants réalisés par d'autres chercheurs canadiens (Brouard, Larivet et Sakka, 2010; Maddil, Brouard et Hebb, 2009; Elson et Hall, 2010).

L'article est divisé en trois sections. La première pose les bases historiques du concept d'entrepreneur et d'entreprise. Elle permet de renouer avec des textes classiques et de rappeler que l'idée d'entreprendre fait l'objet de réflexions théoriques depuis au moins trois siècles. La deuxième section est consacrée à l'étude de l'entrepreneuriat social et collectif. Nous y présentons des éléments de définition, une typologie et les principales caractéristiques de ces types d'entrepreneuriat. La troisième section analyse les informations présentées dans la première et la deuxième section pour dégager dix constats sur l'entrepreneuriat social et 


\section{Fontan (2011)}

collectif. Nous terminons cette section en proposant des critères simples pour une définition de l'entrepreneuriat social et de l'entreprenariat collectif. Enfin, la conclusion se penche sur quelques enjeux et identifie des éléments de perspective permettant de saisir pourquoi il importe de voir l'entrepreneuriat social et l'entreprenariat collectif prendre une plus grande place dans le modèle de développement des économies canadienne et québécoise.

\section{La notion d'entrepreneur}

Le terme « entreprendre » ou encore les notions « d'entrepreneur » ou « d'entreprise » utilisées pour qualifier une activité sociale à vocation économique, sont des termes relativement anciens en langue française, anglaise, allemande et italienne dont le sens moderne prend forme à partir du $18^{\mathrm{e}}$ siècle (Tounes, 2002; Peredo et McLean, 2006).

En langue anglaise, ce terme apparaît une première fois en 1475 pour désigner un manager, un contrôleur, un champion (Oxford English Dictionary, 2003). L'idée plus précise d'associer l'action de contrôle à une action économique émerge au $18^{e}$ siècle. Dans l'Encyclopédie d'Allembert et de Diderot (2010, page Internet), nous retrouvons la définition suivante : « Entrepreneur, c'est celui qui s'engage à faire fabriquer et fournir un vaisseau tout construit, aux termes d'un certain devis qui se fait entre lui et l'acheteur, pour le prix dont ils sont convenus ». Au $18^{\mathrm{e}}$ siècle, l'idée d'entreprendre ou d'entreprise renvoie tant à l'action de produire un ouvrage qu'à celle de faire commerce.

La conception de la fonction d'entrepreneur comme acteur social revient à Cantillon. Ce dernier identifie en 1755 trois groupes d'acteurs présents dans la société française: les propriétaires, indiquant par là des personnes indépendantes de fortune; les fermiers; et les entrepreneurs, c'est-à-dire les marchands, les manufacturiers ou les hommes de loi (Tounes, 2002).

En 1803, Say est le premier économiste à en donner une définition précise : « l'entrepreneur d'industrie, (est) celui qui entreprend de créer pour son compte, à son profit et à ses risques, un produit quelconque ». (Say, 1803, Livre I, p. 66). II s'agit donc d'une personne qui prend des risques afin de produire une valeur économique. Cette production de valeur se fait dans son intérêt et pour son propre bénéfice. Se développe avec Say une conception moderne de l'action économique : il s'agit d'un processus mettant en scène des individus libres qui, afin de s'enrichir et d'enrichir la société (thèse de Smith (1776)), sont amenés à développer un projet d'activité économique productrice d'une richesse qui leur reviendrait de droit étant donné les risques qu'ils ont pris au départ.

Say distingue différents types d'activités sociales: celles permettant la création de richesse économique et qui relèvent de l'entrepreneur d'industrie; celles qui permettent la création de richesse sociale, lesquelles sont prises en charge par des organisateurs de l'entraide ou de la culture par l'entremise des organisations de développement social ou culturel; enfin, celles qui produisent de la richesse politique, donc des actions mises en œuvre par des acteurs de la sphère politique publique ou par des promoteurs d'actions collectives (mouvements sociaux). Say présente ainsi une vision intégrée des actions sociales prenant place dans la société où cette dernière constitue un tout segmenté en grands secteurs d'intervention dédiés au développement économique, au développement social, au développement culturel et au développement politique.

La contribution de Say prépare le travail réalisé par Marshall à la fin du 19e siècle. L'apport de Marshall (1898) est double. D'une part, il étudie le cycle de croissance des entreprises - de la petite entreprise locale à la grande entreprise internationale - et caractérise les possibilités de croissance en fonction de secteurs 


\section{Fontan (2011)}

économiques (certains secteurs étant plus propices que d'autres à leur internationalisation). Au cycle entrepreneurial identifié par Marshall correspond un nouvel acteur : le manager ou le gestionnaire d'entreprise. L'entrepreneur ne représente plus seulement celui qui s'investit par son travail dans la production d'un bien ou d'un service, mais aussi celui qui a pour tâche de gérer les avoirs d'un propriétaire qui a pris une distance et qui délègue la tâche de gestion à un manager.

D'autre part, Marshall observe un phénomène encore peu étudié : l'externalité. D'un côté, l'externalité rend compte de ce qu'une communauté fournit à un entrepreneur. Ce dernier n'a pas à construire en place des routes ou des écoles, cette action incombe à l'État. De l'autre, l'externalité est ce qu'apporte l'entreprise à son milieu. L'entreprise, en produisant des biens, verse des salaires et crée de la richesse locale, laquelle se diffuse sur le territoire en permettant le développement de nouvelles activités (externalité dite positive). Ce faisant, elle génère des irritants, la pollution par exemple (externalité dite négative).

Les réalisations d'une entreprise - externalités positives et négatives - sont la source d'opportunités et rendent compte de situations d'imperfection ou d'incomplétude en ce qui a trait aux modalités de régulation de la société. Les opportunités constituent des occasions d'affaires. Des entreprises sont créées pour offrir des services aux firmes existantes. De telles opportunités permettent la création de nouvelles entreprises, privées ou publiques. Les situations d'imperfection ou d'incomplétude représentent des possibilités d'intervention pour l'État ou pour la société civile et conduisent à la création d'organisations publiques ou sociales. Penser les externalités à la façon d'un moteur permettant une structuration continue des activités socioéconomiques de la société se développera à la suite des travaux de Coase (1960).

En résumé, pour permettre l'entreprenariat, il est nécessaire d'encadrer ce dernier par un environnement culturel qui soit favorable à son émergence et à son développement: l'implantation d'infrastructures (routes, ponts), le développement du capital humain de la population (écoles), et la mise en œuvre de dispositifs pour appuyer le développement de la science et des techniques (recherche et développement en milieux privés ou publics). De façon rétroactive, il s'ensuit un développement des sociétés qui est le résultat de réponses sociales données à des besoins non comblés :

- par le développement d'organisations caritatives, à vocation purement sociale, comme le démontre l'émergence du mouvement moderne des organisations sans but lucratif à vocation sociale dès le début du 19 e siècle;

- par le développement d'un nouveau type d'activités sociales à vocation économique, tel que proposé par différents mouvements sociaux au $19 \mathrm{e}$ siècle, à l'image du mouvement coopératif tel que pensé par les pionniers de Rochdale en Angleterre;

- par l'action interventionniste de l'État, tel qu'en rend compte la synthèse théorique de Keynes (1936).

Notre tour d'horizon sur la notion d'entrepreneur peut difficilement passer sous silence l'apport de Schumpeter (1935). Ses travaux reposent sur l'hypothèse que l'entrepreneur joue un rôle central dans la transformation du système économique moderne. Pour Schumpeter, l'entrepreneur est un innovateur, c'est-à-dire une personne qui, dans et par la réalisation d'une activité économique, est capable de créer de nouveaux arrangements institutionnels, lesquels prennent la forme de nouveaux produits, de nouveaux procédés, de nouveaux modes d'organisation du travail ou de nouveaux usages pour des produits existants. Pour Schumpeter, l'entreprenariat relève d'une action individuelle située et influencée par le contexte social et politique dans lequel elle prend place. Cette action est produite par un être passionné qui cherche continuellement à se dépasser pour trouver 


\section{Fontan (2011)}

les meilleures combinaisons possibles afin de produire de la richesse économique en prenant des risques. L'analyse économique de Schumpeter présente l'entrepreneur comme un bâtisseur dont les actions orientent le développement des sociétés. Schumpeter pose alors une distinction claire entre l'entrepreneur et le gestionnaire, lequel se contente de gérer un actif. Cette distinction attribue un rôle particulier à l'entrepreneur et en fait un cas de figure plus rare. À ses yeux la grande majorité des entreprises sont dirigées par des gestionnaires et moins par des innovateurs. Cette lecture décrit bien la situation économique rencontrée dans les systèmes européens au milieu du $20^{e}$ siècle où le moteur du dynamisme économique est attribué aux actions des grandes firmes, lesquelles sont administrées par des managers.

L'après Deuxième Guerre mondiale est caractérisé par une grande croissance économique dans les pays dits développés (1940-1970). Les turbulences économiques des années 1970 révèlent une situation pour le moins paradoxale (Birch, 1979). Le moteur économique - en ce qui a trait à la création d'emplois - se déplace de la grande firme à la petite et moyenne entreprise. Cette réalité permet de redécouvrir la figure de l'entrepreneur. Aux États-Unis, indique Drucker (1985), entre 1960 et le milieu des années 1980, les grandes firmes perdent environ six millions d'emplois et le secteur public cinq millions alors qu'il se crée environ 40 millions d'emplois au sein de très petites entreprises ou de petites et moyennes entreprises. Dans la même période, des milliers d'organisations sans but lucratif apparaissent 4 . Le dynamisme économique repose dorénavant sur la création de petites unités de production et d'intervention. Tous les regards se tournent alors vers les figures de proue à la gouverne du nouveau moteur économique: l'entrepreneur d'affaires ("business entrepreneur ») et l'entrepreneur social (« social entrepreneur »). Dès lors, les économistes et les grandes écoles de formation redécouvrent la figure de l'entrepreneur et accordent une place centrale à ce dernier pour expliquer les dynamiques de l'économie présentes au début des années 1980 (Julien, 1994; Boutillier et Uzunidis, 1995).

L'exercice que nous venons de faire nous permet de dégager les caractéristiques fondamentales de cette forme d'action sociale que constitue l'entreprenariat. Premièrement, l'entreprenariat met en scène un projet ayant une orientation fondamentalement économique.

Deuxièmement, pour justifier son action, l'entrepreneur invoque une rationalité plus large que la simple recherche du profit. Sans écarter la place importante occupée par la poursuite d'un intérêt personnel, la rationalité d'entreprendre inclut aussi une volonté de réaliser ou de favoriser l'atteinte d'un intérêt plus large.

Troisièmement, entreprendre signifie prendre un risque, investir et aussi mobiliser des ressources et, peut-être, le faire de façon innovante, c'est-à-dire d'une façon qui représente une rupture par rapport aux modes conventionnels de faire.

Quatrièmement, une distinction entre entreprendre et gérer est posée en lien avec l'évolution du cycle de croissance et de développement des entreprises, d'une part, et, d'autre part, des cycles de transformation des systèmes économiques. Cette distinction rend apparente une caractéristique centrale de l'entrepreneur, celle d'être une personne qui innove et dont l'innovation est porteuse de changement au plan économique et surtout qui a un impact à l'échelle de la société.

Cinquièmement, l'étude de l'entreprenariat nous a révélé la présence de deux figures complémentaires.

D'une part, nous retrouvons la figure du grand personnage sur lequel tout repose et sans qui rien n'est possible : l'entrepreneur. II actualise une intuition et met en scène un projet privé qu'il porte de main ferme et qui répond fondamentalement à une vision hiérarchique du fonctionnement de l'entreprise qu'il dirige. $\mathrm{Ce}$ dernier est entouré d'un réseau de ressources, tant sociales que techniques, à la fois tangibles et intangibles. 


\section{Fontan (2011)}

Ce réseau constitue un environnement composé d'une diversité d'éléments qui se moulent et se collent à l'entrepreneur (au leader). Une sorte d'osmose en découle. Elle se traduit par une situation d'accompagnement et parfois même d'encadrement du leader dans la réalisation de son intuition et de son projet. Le projet prend alors une dimension " sociotechnique », au sens où il est soutenu et appuyé pour se matérialiser, se concrétiser, grandir ou tout bonnement disparaître lorsque la résonance sociale et technique autour de ce dernier s'étiole ou que ne peut plus être proprement alimentée la flamme entrepreneuriale du promoteur ou du leader.

D'autre part, les activités économiques suscitent des besoins qui demandent à être comblés par d'autres acteurs, publics, sociaux ou collectifs. À la figure de l'entreprise privée se greffe d'autres types d'entreprenariat dont l'existence même est étroitement associée et imbriquée au système économique dominant. Penser un tel système exige de prendre en considération l'ensemble du portrait.

\section{L'entreprenariat social et collectif}

Aux États-Unis, au Canada, au Québec et en Europe, l'entreprenariat social et l'entreprenariat collectif sont une vieille réalité qui remonte au 19e siècle (Fontan, 1992). L'entreprenariat collectif, sous la forme de coopératives et de mutuelles, a été l'objet d'attentions depuis au moins le début du 20e siècle (MacPherson, 1979; Leikin, 2005; D'Amours, 2006; Petitclerc, 2007). Par contre, aucune notion ne permettait de caractériser l'entreprenariat social, lequel se fondait dans l'action communautaire.

Aux États-Unis, la notion d'entrepreneur social voit le jour à la fin des années 1970. Selon Dees (2001), deux événements qualifient la montée en importance de cette notion. D'une part, en 1980 est créée l'organisation Ashoka. D'autre part, la firme de consultants New Ventures débute ses activités. Ces deux organisations reposent sur deux visions et deux écoles de pensée de ce que représente une entreprise sociale : l'école de l'innovation sociale et l'école de l'entreprise sociale.

Pour Dees (2001), la création d'Ashoka donne naissance à l'école de l'innovation sociale. Cette dernière s'inscrit dans la ligne de pensée de Schumpeter qui lie étroitement le fait d'entreprendre au fait d'innover. L'organisation Ashoka ajoute à la vision de Schumpeter l'idée d'innover non pas pour développer l'économie, mais pour rendre un service public :

Ashoka began by supporting « innovators for the public » or " public entrepreneurs » working internationally. These are people who, according to economic theorist Joseph Schumpeter's description, reform or revolutionize patterns of production. According to Bill, social entrepreneurs were those who were doing just that with regard to socially important goods, like education and healthcare and access to credit. (Fulton et Dees, 2006, p. 4)

Toujours selon Dees (2001), la création de New Ventures marque les débuts de l'école de l'entreprise sociale. En 1980, il s'agissait pour Skloot, créateur de cette dernière, de répondre à des demandes qui lui étaient adressées par des organisations à but non lucratif. Confrontées à des problèmes de financement, ces dernières devaient trouver de nouvelles sources de revenus : « In the United States, federal and state funding for nonprofits decreased $23 \%$ in the 1980s, and continued to decline in the 1990s " (Johnson, 2000, p. 3). Selon Skloot, il fallait profiter du potentiel et de la réalité d'une organisation vouée au développement social ou au développement politique afin de générer des revenus qui lui permettraient de combler la perte ou l'absence de revenus provenant de fondations privées ou de l'État : 


\section{Fontan (2011)}

Ed was concerned with helping nonprofits find new streams of revenue to make them more financially viable. This evolved into helping nonprofits use business ideas and tools more generally. (Dees, 2001, p. 4)

Ces deux écoles représentent deux philosophies de travail. Ashoka œuvre au développement de leaders sociaux par la formation d'un vaste réseau d'entrepreneurs sociaux. La logique de travail est d'identifier et de soutenir pendant trois ans la formation, le coaching et le parrainage de créateurs d'entreprises ayant la conscience de militants sociaux (Defourny, 2006). New Ventures œuvre au développement de marchés pour des organisations sociales en les amenant à comprendre et à utiliser les outils classiques de développement et de gestion de projets utilisés par des entrepreneurs privés.

Au début des années 1980, l'engouement pour l'entreprenariat social est bien compris et pris en compte par différentes universités états-uniennes. Des cours se développent et des programmes sont implantés à partir de la fin des années 1980 et au début des années 1990 : création d'une formation en entreprenariat à l'Université de Washington, mise sur pied de la Social Enterprise Initiative en 1993 à l'Université Harvard, du Center for the Advancement of Social Entrepreneurship à l'Université Duke ou encore du Center for Social Innovation à I'Université Stanford (Defourny, 2006; Fulton et Dees, 2006).

Au Canada, l'entreprenariat social est une réalité plus récente. Moins nombreux qu'aux États-Unis sont les chercheurs qui ont réalisé des travaux sur cette question ${ }^{5}$. Le faible développement de recherches sur ce thème tient, selon Johnson, à un malaise présent au sein de la communauté canadienne face au concept de l'entreprise sociale: " The concept and practice of social entrepreneurship has been widely embraced in the U.S. and the U.K., but has met with less enthusiasm in Canada » (Johnson, 2003, p. 5).

En fait, la pratique était autant présente en sol canadien qu'ailleurs dans le monde; toutefois, cette pratique ne faisait pas l'objet d'une reconnaissance spécifique de la part du milieu académique. II existait plusieurs concepts apparentés, par exemple ceux d'entreprises communautaires et d'entreprises d'économie sociale (coopératives et mutuelles). Ces termes regroupaient largement les pratiques couvertes par les définitions états-uniennes de l'entreprise sociale.

Depuis une décennie, la situation s'est passablement transformée. En effet, plusieurs organisations, dont des fondations privées et des centres universitaires, travaillent maintenant au développement de l'entreprise sociale ou du leadership socioéconomique, dont les Fondation Muttart, McConnell, le Fraser Valley Centre for Social Enterprise, le Centre Sprott pour les entreprises sociales, le Canadian Centre for Social Entrepreneurship et le Enterprising Non-Profits. Preuve de l'importance du concept, cette dernière organisation réalise en janvier 2007 à Vancouver un premier colloque national (Canadian Conference on Social Enterprise) sur le thème de l'entreprenariat social. Enfin, une définition large de l'entreprenariat social est proposée par le Centre Sprott :

Social enterprises are defined as organizations created to pursue social missions or purposes that operate to create community benefit regardless of ownership or legal structure and with various degrees of financial self-sufficiency, innovation and social transformation. (Brouard, Hebb et Madill, 2008, p. 11)

Au Québec, il nous faut mentionner le travail réalisé par Lévesque (2002). Celui-ci indique que la poursuite d'activités à vocation économique est l'œuvre d'au moins trois types d'entrepreneurs - l'entrepreneur privé, l'entrepreneur social et l'entrepreneur collectif - qu'il distingue et caractérise à partir d'un ensemble 
d'indicateurs (tableau 1). À ces trois figures, nous greffons une quatrième, celle de l'entrepreneur public caractérisé par les sociétés d'État (Bernier et Fortin, 1997).

Lévesque présente une synthèse éclairante sur les différents types d'entreprenariat en raison des éléments comparatifs qu'il identifie. Prendre des risques, innover et se donner un projet d'entreprise sont des points communs entre l'entrepreneur privé et l'entrepreneur social ou collectif. La distinction entre l'entrepreneur privé (EP) et l'entrepreneur social (ES) ou collectif (EC) repose fondamentalement sur la nature du projet développé par le ou les promoteurs :

- II y a des risques, mais ils ne sont pas du même ordre puisqu'ils ne sont pas assumés pas un individu ou un groupe à des fins privées (EP) mais par un groupe à des fins sociales ou collectives (ES ou EC);

- Les moyens mobilisés (ES et EC) sont différents de l'entreprise privée puisqu'ils impliquent une mobilisation large de ressources issues de la communauté et de l'État dans une perspective dépassant une finalité purement économique à des fins privées;

- Le projet qui est proposé combine, sous un mode de gestion démocratique, des objectifs économiques et des objectifs sociaux auxquels s'ajoutent parfois, selon les recherches que nous avons effectuées (Fontan, Leblanc, Noiseux et Silvestro, 2010), des objectifs écologiques.

Tableau 1 : Typologie des formes d'entreprenariat

\begin{tabular}{|c|c|c|c|c|c|}
\hline Dimension & $\begin{array}{c}\text { Individu } \\
\text { Collectif } \\
\text { Communauté }\end{array}$ & Rationalité & Risque & Projet & Innovation \\
\hline Privé & Plutôt individu & $\begin{array}{c}\text { Plutôt formel } \\
\text { (calcul comptable) }\end{array}$ & Financier & $\begin{array}{c}\text { Plutôt individu } \\
\text { (réalisation de soi } \\
\text { et famille) }\end{array}$ & $\begin{array}{c}\text { Sens } \\
\text { schumpétérien }\end{array}$ \\
\hline Social & Individu & $\begin{array}{c}\text { Plutôt en valeur } \\
\text { vers la } \\
\text { communauté } \\
\text { (proximité) }\end{array}$ & $\begin{array}{c}\text { Financier et social } \\
\text { (réputation) } \\
\text { Risque moindre }\end{array}$ & $\begin{array}{c}\text { Plutôt engagement } \\
\text { social } \\
\text { Développement } \\
\text { local (intérêt } \\
\text { général) }\end{array}$ & $\begin{array}{c}\text { Besoins non } \\
\text { satisfaits + formes } \\
\text { d'organisation }\end{array}$ \\
\hline Collectif & Individu & $\begin{array}{c}\text { Plutôt en valeur } \\
\text { vers le collectif } \\
\text { (membres) }\end{array}$ & $\begin{array}{c}\text { Financier et social } \\
\text { (réputation) } \\
\text { Risque moindre }\end{array}$ & $\begin{array}{c}\text { Plutôt } \\
\text { entrepreneurial } \\
\text { mais collectif }\end{array}$ & $\begin{array}{c}\text { Besoins non } \\
\text { satisfaits + formes } \\
\text { d'organisation }\end{array}$ \\
\hline
\end{tabular}

Source : Lévesque, 2002, p. 20

Tel qu'en rend compte la synthèse de Lévesque, l'entreprenariat privé est un projet motivé par des intérêts personnels d'enrichissement et d'accomplissement de soi à partir d'un modèle vertical et hiérarchique de gouvernance de l'entreprise s'appuyant sur une propriété privée des avoirs générés.

L'entreprenariat social (ES) et l'entreprenariat collectif $(E C)$ reposent sur des projets visant l'enrichissement et l'accomplissement d'une communauté à partir d'intérêts sociaux (ES) ou collectivisés (EC) qui sont coordonnés à l'aide d'un modèle horizontal et vertical de gouvernance et d'une propriété dite sociale (ES) ou collective (EC). Les projets qu'ils soutiennent impliquent une production de valeur économique et sociale (Bassi, 2011) partagée entre les membres du groupe et la communauté. 


\section{Fontan (2011)}

L'entreprenariat - social et collectif - se distingue de l'entrepreneuriat privé par le fait qu'il met en scène des projets communautaires ou collectifs. II est motivé par une production de valeur vouée au développement $d u$ bien-être d'un groupe ou d'une communauté. II repose sur un mode de gouvernance communautaire ou collectif, où le sens donné à la notion de communauté peut être large ou restreint.

En Europe, à partir des années 1970, émerge une forme collective d'entreprenariat social. Un premier développement est observable en Italie. II est associé à la création d'un nouveau type de coopérative : les coopératives de solidarité sociale. Ces coopératives offrent des services sociaux ou facilitent l'insertion de leurs membres sur le marché du travail, à l'image des coopératives sociales d'insertion (Borzaga et Santuari, 1998).

Des travaux importants ont été réalisés par le European Research Network. Ce groupe, dirigé par Defourny (2006), a réalisé une étude très importante sur l'émergence des entreprises sociales européennes pour une période s'étendant entre 1996 et 2000. Bien que l'Europe n'ait pas développé une politique spécifique pour les entreprises sociales, on y dénombre plusieurs politiques sectorielles pour appuyer le développement du tiers secteur et de l'économie sociale.

Afin d'appuyer le développement de l'entreprenariat social, le Programme Leonardo da Vinci (2002) de la Commission européenne a développé un référentiel sur le métier d'entrepreneur social. Cette organisation identifie nommément un métier et un champ de compétences pour qualifier les qualités de l'entreprenariat et de l'entrepreneur social. Le référentiel vise le développement du capital social de futurs entrepreneurs sociaux en offrant des formations sur un ensemble de thématiques. II propose une définition engagée de l'entreprenariat social et s'est donné pour objectif de faciliter la reconnaissance sociale de cet entreprenariat :

L'entrepreneur social exerce une fonction de direction dans une entreprise dont la finalité économique est conjointe ou subordonnée à une finalité répondant à des valeurs de solidarité entre personnes, groupes sociaux, territoires.

Son action est de concevoir, promouvoir, développer l'activité de cette entreprise dans sa finalité solidaire et sociale.

En aucun cas la recherche du profit n'est le critère exclusif de ses choix d'orientation ou de gestion même si à certains moments la recherche de l'équilibre économique tend à orienter prioritairement mais provisoirement les choix. Lorsqu'un patrimoine collectif est constitué (exemple des coopératives), sa gestion est également collective (une personne, une voix). (Programme Leonardo da Vinci, 2002, p. 18)

Au Royaume-Uni, l'entreprenariat social a été reconnu par le législateur public au début de l'année 2000. Ce dernier a développé une politique d'appui et de soutien à l'entreprenariat social. Le gouvernement du RoyaumeUni s'est donné une double définition de l'entreprenariat social. Cette dernière inclut principalement des entreprises sociales tout en accordant une place à certaines entreprises coopératives.

A social enterprise is a business with primarily social objectives whose surpluses are principally reinvested for that purpose in the business or in the community, rather than being driven by the need to maximize profit for shareholders and owners. (Cabinet Office, 2006, p. 10) 


\section{Fontan (2011)}

Les entreprises sociales sont considérées comme étant partie prenante du troisième secteur, en complémentarité des entreprises privées et publiques.

Social enterprises are part of the "third sector", which encompasses all organizations which are non-governmental, principally reinvest surpluses in the community or organization and seek to deliver social or environmental benefits. The third sector embraces voluntary and community organizations, charities, social enterprises, mutuals and co-operatives ». (Cabinet Office, 2006, p. 10)

A co-operative is defined as an autonomous association of persons united voluntarily to meet their common economic, social and/or cultural needs and aspirations through a jointly owned and democratically controlled enterprise. (Cabinet Office, 2006, p. 11)

Le portait que nous avons réalisé par cette revue de littérature de l'entreprenariat - social et collectif - nous a permis d'observer à quel point cette pratique est présente en Amérique du Nord et en Europe. Elle regroupe des projets poursuivant des finalités relativement convergentes sans pour autant correspondre tout à fait à la même réalité.

\section{CONSTATS}

\section{Le tour d'horizon que nous avons effectué nous permet de poser un ensemble de constats.}

Premièrement, il est reconnu tant en Amérique du Nord qu'en Europe que la pratique de l'entreprenariat collectif est ancienne alors que l'entreprenariat social est une nouvelle notion qui apparaît à la fin des années 1970. À travers une terminologie éclatée - entreprise sociale, entreprise collective, société à utilité ou à finalité sociale, entreprise solidaire, etc. - la diversité des actions qu'il suscite, les ressources qu'il mobilise et l'implication à son égard de la part des législateurs publics font en sorte que la pratique de l'entreprenariat social a acquis une légitimité et une crédibilité à l'échelle internationale.

Deuxièmement, à partir des années 1980, les États-Unis sont au cœur du processus de reconnaissance des entreprises sociales. Le volet " entreprise collective », généralement associé au secteur de l'économie sociale ou solidaire, connaît quant à lui un renouveau important au cours de la même période en Italie, en France et au Canada.

Troisièmement, le processus de reconnaissance de l'entreprenariat social est fondamentalement lié aux transformations qui touchent les pays développés. À partir des années 1980, les transformations liées à la perte d'importance des grandes firmes comme moteur économique de la croissance des économies développées et la montée en importance de très petites, petites et moyennes entreprises ou organisations, d'une part, et, d'autre part, la réduction des financements publics consacrés à des organisations sans but lucratiff, sont au cœur du renouveau de ce mode entrepreneurial. Dans les travaux consultés, ces transformations sont aussi liées au désir et à la volonté d'acteurs sociaux d'investir le secteur de la société civile et la forme juridique de l'organisme sans but lucratif afin de trouver des solutions alternatives aux incapacités du marché et de l'État à répondre de façon adéquate aux urgences sociales.

Quatrièmement, l'entreprise sociale est une réalité hétérogène. Les travaux consultés nous permettent de dire que l'étude, la promotion et le soutien offerts aux entreprises sociales poursuivent deux objectifs. D'un côté, des efforts, sous forme de ressources, sont mobilisés pour assurer le soutien à des organisations. L'accent est placé sur le développement de l'entreprise sociale, d'un groupe d'entreprises, d'un secteur ou d'une filière. II 


\section{Fontan (2011)}

s'agit alors de travailler sur les différentes phases de ce type d'entreprenariat : de l'émergence à l'expansion en passant par la consolidation des activités. D'un autre côté, des efforts, sous forme de ressources, sont concentrés sur le soutien à apporter pour favoriser l'émergence de leaders sociaux au sein de différentes communautés ou différents territoires, et ce indépendamment du développement d'une organisation spécifique.

L'analyse des travaux sur l'entreprenariat non privé permet de comprendre que le continuum des cas de figure rencontrés est très large. II peut s'agir :

- d'un projet ou d'un volet social au sein d'une entreprise privée ou d'une organisation publique;

- d'un nouveau marché à vocation sociale qui est pris en charge par des entreprises privées (les services privés de développement de l'employabilité par exemple);

- d'une action à vocation économique développée par un organisme sans but lucratif pour consolider son financement;

- d'initiatives mises de l'avant, par un collectif ou une communauté, dans un secteur particulier ou sur un territoire donné, à partir d'organismes sans but lucratif pour développer de façon différente le marché et l'économie : champs de l'action communautaire, de l'action environnementale, du commerce équitable, de l'agriculture soutenue par la communauté, de l'insertion par l'économique, de l'économie sociale ou solidaire, du développement local ou de la coopération solidaire à l'international;

- d'une initiative mise de l'avant par un collectif à partir d'une organisation coopérative ou mutualiste pour développer un marché et une économie plus démocratique et solidaire.

Ceci permet de dire qu'il existe trois grandes familles de cas pour qualifier l'entreprenariat social et l'entreprenariat collectif :

- les initiatives purement privées à vocation sociale, mais distinctes de celles représentées par le concept de « responsabilité sociale des entreprises »;

- les initiatives communautaires ou humanitaires développant des marchés à vocation sociale;

- enfin, les initiatives collectives - coopératives, mutuelles, organisations autogestionnaires ou autonomes - qui sont dotées d'une mission sociale, communautaire ou humanitaire.

Somme toute, une distinction centrale est évoquée par de nombreux travaux pour qualifier tant la réalité de l'entrepreneur social que celle de l'entrepreneur collectif. Cette distinction fait actuellement l'objet d'un débat et regroupe principalement des analyses critiques. Elle associe fondamentalement l'entreprenariat social ou collectif à des valeurs liées à la recherche de solidarité, de justice et d'équité. II est question d'une éthique sociale, de principes communautaires et d'une vision solidaire du développement à effectuer.

Il existe un autre débat sur le fait que l'entreprise sociale ou l'entreprise collective puissent ou non générer des profits en réalisant sa mission. Tous les travaux mentionnent le fait que les entreprises - sociales et collectives - doivent apporter une valeur ajoutée sociale à une communauté, à une collectivité ou à un territoire. Par 


\section{Fontan (2011)}

contre, il n'y a pas de consensus sur le fait que, pour y arriver, il faille fondamentalement accumuler des bénéfices. Cette question est particulièrement présente lorsque l'État contractualise des ententes avec des entreprises sociales ou des entreprises collectives. L'achat de services qu'il fait couvre essentiellement les frais courants. L'État-patron ne valorise et ne permet pas la production d'un « surplus ».

Enfin, l'analyse nous permet d'affirmer - peu importe les cas de figure rencontrés - que l'État est concerné à plusieurs niveaux par le développement de ces formes d'entreprenariat. D'une part, l'État est responsable de la conduite d'actions de règlementation visant le développement social, culturel, politique ou économique de la société en général, et le développement de communautés et de territoires en particulier. II l'est à titre de régulateur en raison du fait qu'il lui est demandé de mettre en place un cadre institutionnel adéquat au moyen de politiques, de programmes, de services, de compétences et d'attitudes. L'État est présent aussi à titre de facilitateur. II lui est demandé de dégager les ressources nécessaires pour appuyer l'émergence, la consolidation et l'expansion de toutes les formes d'entreprenariat.

Enfin, l'État est concerné à un autre niveau : en tant qu'acteur participant directement au développement prenant place sur son territoire. Dans l'administration publique, de l'échelle fédérale jusqu'à la municipale, il pourrait se créer des initiatives parapubliques à finalité socioéconomique et à portée sociale. Du monde de l'éducation à celui de la valorisation des ressources humaines en passant par les actions publiques de développement économique (habitat, santé, transport, environnement, etc.), le domaine public pourrait initier des projets d'entreprenariat social à vocation publique.

Cinquièmement, nous observons peu de travaux sur les qualités inhérentes ou naturelles de l'entrepreneur ou du leader responsable du développement d'une entreprise, qu'elle soit sociale ou collective. Le débat actuel porte essentiellement sur les actions à mener pour assurer un bon milieu d'incubation afin que puisse se développer un nombre suffisant d'entrepreneurs sociaux et collectifs.

Dans la littérature consultée, le développement de l'entreprise - sociale ou collective - est envisagé sous forme d'occasions à saisir dans un contexte où il incombe à l'État et à des organisations de la société civile de mettre en place un environnement qui soit favorable à ce type d'entreprenariat. II est aussi indiqué le besoin d'assurer une durabilité à ces formes entrepreneuriales. II s'agit donc d'implanter les conditions pour innover dans la mise en place de mesures d'aide ou de soutien aux entrepreneurs sociaux et aux entrepreneurs collectifs.

De plus, il est fréquemment évoqué que le cadre de développement de cet entreprenariat est très lié au contexte social dans lequel il prend forme. II est donc développé en fonction des caractéristiques démographiques, sociales, économiques, culturelles ou ethniques des populations ou des territoires concernés. Dès lors, l'entreprenariat - social et collectif - cumule différentes formes de capital : humain, social et socioterritorial (Tremblay, Klein et Fontan, 2009).

Les travaux consultés nous indiquent que la mobilisation de ressources nécessitées pour l'implantation de ces types d'entreprenariat se fait en interpellant un nombre important de secteurs de la société. Autant le capital privé et l'État que la société civile, y compris les mouvements sociaux, sont invités à mettre à la disposition des entrepreneurs concernés leurs ressources pour faciliter le développement de l'entreprenariat social ou de l'entreprenariat collectif.

Sixièmement, les travaux rendent compte d'un consensus : l'entreprenariat social et l'entreprenariat collectif sont sources d'innovation sociale. Ces formes entrepreneuriales permettent de faire des choses de façon différente. Elles facilitent l'adoption de solutions là où l'action privée traditionnelle et l'action publique classique 


\section{Fontan (2011)}

ne peuvent ou ne veulent pas intervenir. Ces formes d'entreprenariat se révèlent être une façon d'ouvrir les horizons et de faire reculer des frontières socioéconomiques ou sociopolitiques.

Somme toute, il existe des différences notables en ce qui a trait aux finalités des innovations développées par ces deux types d'entreprenariat. À titre indicatif exploratoire et non exhaustif, ces finalités sont partagées entre :

- des actions réformistes à portée mineure [innovation de dissémination d'une nouveauté (nouveau service)] ou à portée majeure [innovation mettant en scène une nouveauté importante (nouveau marché; nouvelle organisation)];

- des actions dites de rupture à portée stratégique liée à la dissémination d'une nouvelle stratégie (nouvelle organisation du travail ou nouveau mode de gouvernance) ou à portée radicale (innovation qui met en place un nouveau système économique ou politique).

Septièmement, nous avons observé une certaine zone de tension entre des définitions économiques de l'entreprise sociale à vocation sociale et des définitions plus politiques de l'entreprise collective à vocation économique. II existe donc des visions divergentes sur le type de système économique qui est souhaité ou promu par les entrepreneurs sociaux et les entrepreneurs collectifs. Là encore une classification se dégage entre des actions :

- proposant un capitalisme à visage humain :

○ rendant légitime l'existence d'organisations vouées au développement social, leur permettant d'acquérir plus d'autonomie en les habilitant aux méthodes entrepreneuriales traditionnelles;

- favorisant le développement d'une économie de marché plus démocratique;

- proposant un changement radical de système économique qui conduirait à une transformation en profondeur des arrangements institutionnels des sociétés modernes.

Huitièmement, à partir de ces nouvelles formes d'entreprenariat, il se construit un nouveau champ d'expertise et de compétences, de nouveaux métiers en quelque sorte, lesquels exigent une adaptation des curriculums de formation, d'éducation professionnelle et d'éducation supérieure. II est aussi dit que les compétences exigées par le métier d'entrepreneur social et d'entrepreneur collectif sont encore mal définies et demandent à être mieux identifiées à partir d'études ou d'enquêtes qui porteraient sur des populations plus larges que celles qui ont été recensées jusqu'à présent. La plupart des études que nous avons repérées reposent sur un petit échantillon d'organisations et sur des entrevues réalisées auprès d'un petit nombre d'entrepreneurs.

Neuvièmement, trois grandes stratégies de promotion de cet entreprenariat sont mentionnées :

- Une première stratégie, dite décentralisée, est fortement liée aux actions développées par des entreprises sociales (fondations ou organisations de consultants sociaux) ou par des organisations d'enseignement supérieur (universités et collèges) - une stratégie très présente aux États-Unis. 
- Une deuxième stratégie, dite centralisée, est associée au travail développé par le législateur public national pour doter le pays d'une législation, de ressources et d'un cadre public d'intervention en entreprenariat social (le cas du RoyaumeUni).

- II y a en outre une troisième stratégie, que l'on qualifie de mitoyenne, où des actions à la pièce sont réalisées par différents niveaux de gouvernement, des mouvements sociaux, des fondations, des entreprises sociales, solidaires ou collectives (le cas de l'Europe).

Dixièmement, et en guise de conclusion de cette section, nous sommes en mesure d'identifier des critères relativement simples pour distinguer l'entreprenariat social et l'entreprenariat collectif des autres formes d'entreprenariat. Ces critères rendent compte de la grande hétérogénéité des situations entrepreneuriales existantes ou à venir. Ils ont l'avantage d'être inclusifs sans être larges au point où toute action sociale qui serait menée par une entreprise ou une organisation ferait de celle-ci soit une entreprise sociale soit une entreprise collective. Ces critères sont les suivants :

- fonctionnement démocratique de l'entreprise, de la coopérative, de la mutuelle ou de l'organisme sans but lucratif;

- ancrage ou arrimage communautaire bien constitué;

- combinaison d'objectifs sociaux et économiques, lesquels doivent être encastrés dans une mission socioéconomique de développement d'un groupe ou d'une communauté qui est définie sur une base sectorielle ou territoriale;

- volonté de garder une autonomie forte par rapport à l'État;

- prise en compte émergente d'objectifs environnementaux.

\section{CONCLUSION}

Le Canada, à l'image des autres pays membres de l'OCDE, est confronté à trois grands enjeux directement liés à l'émergence d'une nouvelle question mondiale. Premièrement, malgré des avancées importantes dans la façon de générer et de redistribuer la richesse économique, force est de constater que les retombées sous forme d'enrichissement social n'évoluent pas à une vitesse aussi rapide que les retombées de type richesse économique cumulée par des familles, des individus ou des groupes corporatifs (Yalnizyan, 2007). Dès lors, un écart grandissant sépare la grande richesse de la grande pauvreté.

Deuxièmement, le cadre même du vivre ensemble et de la création de richesse est confronté au grand défi de la dégradation des systèmes écologiques. Une expression de cette dégradation a pris la forme du spectre très médiatisé que représente le réchauffement de la planète et les conséquences désastreuses anticipées de ce réchauffement sur les communautés humaines.

Troisièmement, le développement social de la société canadienne repose de plus en plus sur un nouveau contrat social devant tenir compte de la diversité culturelle et de nouvelles exigences sociales. Ces exigences s'expriment de façon diverse sous forme de décentralisation et de participation citoyenne, de démocratie locale, de diversité culturelle, de diversité sexuelle, etc. (Fontan, 2011). 


\section{Fontan (2011)}

Ces trois éléments se combinent et exercent des pressions sur les organisations et les institutions canadiennes. Concrètement, ces enjeux indiquent des besoins non comblés, des urgences à rencontrer, des exigences et des aspirations à prendre en considération. Les organisations et les institutions sont questionnées et sont appelées, pour satisfaire aux nouvelles demandes portées par la population, à s'adapter à cette réalité et à le faire dans la concertation et la mise en synergie des ressources qu'elles mobilisent.

L'entreprise sociale et collective, eu égard aux enjeux nommés précédemment et dans la veine des traditions issues des mouvements sociaux, de l'action communautaire et de l'économie sociale, offre une capacité d'innovation plus grande que l'entreprise classique. Ces formes entrepreneuriales ont une plus grande facilité d'innovation sociale parce qu'elles ont la capacité de reconnaître le droit de parole, de participation ou de gérance à un ensemble de parties prenantes qui peuvent directement être impliquées dans la solution des problèmes rencontrés ou dans la réalisation des aspirations envisagées (Klein et Harrisson, 2007). L'enjeu évidemment est de permettre cette démocratisation. Nos travaux nous ont permis d'observer que dans certains contextes de revitalisation territoriale où des actions de lutte contre la pauvreté et l'exclusion portées par des entreprises sociales et des entreprises collectives sont en mesure de tenir compte de la volonté de citoyens de s'impliquer et de participer à la résolution des problèmes rencontrés au sein de leur communauté (Klein, Fontan, Saucier, Tremblay, Tremblay et Simard, 2009).

C'est donc dans la capacité d'établir un dialogue avec les citoyens et les citoyennes que ce type d'entreprenariat se révèle être un outil approprié pour réaliser un projet socioéconomique à portée politique centré sur la combinaison d'objectifs sociaux, économiques et environnementaux. C'est dans et par cette capacité d'écoute et d'empathie que se distingue, pour des entrepreneurs sociaux ou des entrepreneurs collectifs, leur choix de s'investir dans l'entreprenariat social ou dans l'entreprenariat collectif plutôt que dans l'entreprenariat classique, tout en le faisant sans tomber dans le piège d'une professionnalisation éteignoir. En résumé, cet entreprenariat permet, lorsque les conditions sociopolitiques sont bien réunies, d'internaliser différents types d'enjeux et d'innover par des rapports sociaux qui sont appelés à être plus :

- solidaires relativement à la question de la création-redistribution de la richesse;

- démocratiques relativement aux projets et à la participation de différents publics;

- respectueux envers la diversité culturelle et les différences de genre;

- écologiques par rapport à la viabilité et la durabilité des systèmes environnementaux.

Certes, toutes les entreprises sociales et toutes les entreprises collectives n'ont pas le même potentiel réformateur ou de rupture et toutes n'embrassent pas l'ensemble de ces enjeux.

L'actualisation, avec plus ou moins d'accent, de l'un ou l'autre de ces enjeux repose sur la qualité du projet politique et la quantité des ressources mobilisées au sein et autour de l'entreprise, qu'elle soit sociale ou collective. La portée de leurs actions dépend certainement de la qualité des relations qu'elles tissent avec leur communauté et de la nature, bienveillante ou malveillante, de l'environnement institutionnel dans lequel elles se déploient. II importe donc de reconnaître à leur juste valeur tant les limites que les potentialités de ces formes d'entreprenariat. 


\section{Fontan (2011)}

\section{NOTES}

1. Voir les textes de : MacPherson (1979), Vanek (1989), Fontan (1992), Johnson (2000, 2003), Lévesque (2002), Williams (2005), Restakis (2006), Riverin (2006), Painter (2006), Peredo et McLean (2006), Gould (2006), Brouard (2006), Brouard et al. (2009), Elson et Hall (2010) et Manwaring et Valentine (2011).

2. Voir les textes de : Ashoka (non daté), Melville et Walesh (1997), Dees (1998), Thompson, Alvy et Lees (2000), Dees (2001), Alter (2004-2006), Robinson et Lo (2005), Alvord, Brown et Letts (2005), Kramer (2005), Fulton et Dees (2006), Harding (2006), Ryzin et Lévy et Rival (2010).

3. Voir les textes de : Defourny (1994), Borzaga et Santuari (1998), Clément et Gardin (2000), Defourny et Nyssens (2001), Lavillet et Nyssens (2001), Tounes (2002), Boncler et Hlady-Rispal (2003), Casson (2005), Spear (2006), Defourny (2006), Bassi (2009), Van Ryzin, Grossman, Sipadova-Stocks et Bergrud (2009), Bacq et Janssen (2010) et Trivedi (2010ab, 2011).

4. «Between 1977 and 1997, the revenues of America's non-profit organizations increased by 144 percent after adjusting for inflation, nearly twice the growth rate of the nation's economy. Especially robust was the revenue growth among arts and culture, social service, and health organizations. Not only non-profit revenues but also the number of organizations has grown substantially. Between 1977 and 1997 the number of $501(c)(3)$ and $501(c)(4)$ organizations registered with the Internal Revenue Service increased by 115 percent, or about 23,000 organizations per year. This compares with a 76 percent growth rate among for-profit businesses » (Salamon, 2002, p. XV).

5. Mentionnons les travaux de Johnson (2000 et 2003), Lévesque (2002), Peredo et McLean (2006), Brouard (2006), Audet et Julien (2006), Riverin (2006), Anderson, Dana et Dana (2006) et Brouard et al. (2009).

6. II est à noter qu'au Québec l'État maintient et même augmente sa participation au financement d'organismes sans but lucratif. II s'agit d'une situation très différente de celle rencontrée aux États-Unis à partir des années 1980 où l'État réduit de façon très importante sa participation au financement de ces organisations (Jetté, 2008).

7. Par responsabilité sociale des entreprises, nous retenons la définition de la Commission européenne: "Le concept de responsabilité sociale des entreprises signifie essentiellement que celles-ci décident de leur propre initiative de contribuer à améliorer la société et rendre plus propre l'environnement » (Commission européenne, 2001, p. 5).

\section{REFERENCES / BIBLIOGRAPHIE}

Alter, K. (2004-2006). « Social Enterprise Typology », Virtue Ventures. URL : http://virtueventures.com/setypology [1 mai 2010].

Alvord, S.H., Brown, D.L., Letts, C.W. (2004). Social Entrepreneurs and Social Transformation: An Exploratory Study. The Hauser Center for Nonprofit Organization and The Kennedy School of Government, Harvard University.

Anderson, R.B., Dana, L.P., Dana, T.E. (2006). «Indigenous land rights, entrepreneurship, and economic development in Canada: "Opting-in" to the global economy ». Journal of World Business, 41(1), 45-55.

Ashoka (non daté). The State of Social Entrepreneurship Learning. A survey of course work and extracurricular activity. The Global Academy for Social Entrepreneurship at Ashoka. Innovators for the Public, 1-42.

Audet, J., Julien, P.A. (2006). « L'entrepreneuriat social au Québec. L'exemple des Centres de formation en entreprise et récupération ». Recherches sociographiques, XLVII(1), 69-94. 


\section{Fontan (2011)}

Bacq, S., Janssen F. (2011). " Structuring the Field of Social Entrepreneurship: A Transatlantic Comparative Approach ». In Borch, O.J., Fayolle, A., Kyrö, P., Ljunggren. E. (eds.). The Entrepreneurship Research in Europe: Evolving Concepts and Processes. Cheltenham-Northampton, Edward Elgar Publications.

Bassi, A. (2011). « II valore sociale aggiunto delle organizzazioni di terzo settore che erogano servizi alla persona. Verso un sistema di indicatori (VSA) per la misurazione della performance delle imprese sociali ». In Donati, P., Colozzi, I (a cura di). « II valore aggiunto delle relazioni sociali, numero monografico della rivista ». Sociologia e Politiche Sociali, V(1).

Bassi, A. (2009). Social entrepreneur, social entrepreneurship, social entreprise. Three terms in quest of a theoretical framework. Trento, Italy. Article présenté au 2nd EMES International Conference on Social Enterprise, 1-4 July.

Bernier, L., Fortin, J.P. (1997). « L'entrepreneurship public dans les sociétés d'État au Québec ». Revue organisation, 6(1), hiver, 13-24.

Birch, D. (1979). The Job Generation Process. Cambridge (Mass.). MIT Press.

Boncler, J., Hlady-Rispal, M. (2003). Caractérisation de l'entrepreneuriat en économie sociale, Rimouski, Éditions de l'ADREG.

Borzaga, C., Santuari, A. (eds.). (1998). Social enterprises and new employment in Europe. Autonome Region Trentino-Südtirol. University of Trento.

Bouthillier, S., Uzunidis, D. (1995). L'entrepreneur: une analyse socio-économique. Paris. Éditions Économica.

Brouard, F. (2006). L'entrepreneuriat social, mieux connaitre le concept. Trois-Rivières, 23e Colloque annuel of The Canadian Council for Small Business \& Entrepreneurship.

Brouard, F., Hebb, T., Madill, J. (2008). A Typology of Social Enterprise, Ottawa. Carleton Centre for Community Innovation. WP 09-01.

Brouard, F., Larivet, S, Sakka, O. (2010). "Entrepreneuriat social et participation citoyenne». ANSERJ - Revue canadienne de recherche sur les OSBL et l'économie sociale / Canadian journal of nonprofit and social economy research, 1(1), 46-64.

Bull, M. (2008). « Challenging tensions: critical, theoretical and empirical perspectives on social enterprise ». International Journal of Entrepreneurial Behaviour \& Research, 14(5), 268-275.

Cabinet Office. (2006). Social Enterprise Action Plan, Scaling New Heights. London. Office of the Third Sector.

Casson, Mark. (2005). «Entrepreneurship and the theory of the firm ». Journal of Economic Behaviour and Organization, 58, 327-348.

Clement, H., Gardin, L. (2000). L'entreprise sociale. Les notes de l'Institut Karl Polanyi. Thierry Quinqueton Éditeur.

Coase, R.H. (1960). « The Problem of Social Cost ». Journal of Law and Economics, 3, 1-44.

Commission européenne. (2001). « Promouvoir un cadre européen pour la responsabilité sociale des entreprises ». Bruxelles. Livre vert, direction générale de l'Emploi et des Affaires sociales, unité EMPL/D.1.

D’Amours, M. (2006). L'économie sociale au Québec. Montréal. Éditions St-Martin.

Dees, J.G. (2001). The Meaning of Social Entrepreneurship, Graduate School of Business, Stanford University. URL: www.caseatduke.org/documents/dees_sedef.pdf, 3 février 2010.

Defourny, J. (2006). L'émergence de l'entreprise sociale: concepts et réalités. Université libre de Bruxelles. Chaire Franqui.

Defourny, J. (Dir.). (1994). Développer l'entreprise sociale. Bruxelles. Fondation Roi Baudouin.

Defourny, J., Nyssens, M. (2001). « Belgium: Social Enterprises in Community Services ». In Borzaga, C., Defourny, J. (Eds). The Emergence of Social Enterprise. Londres, Routledge, 47-64. 


\section{Fontan (2011)}

Draperie, J.F. (2010). «L'entrepreneuriat social, un mouvement de pensée inscrit dans le capitalisme ». Revue internationale d'économie sociale. URL : http://www.recma.org/node/974. [10 août 2010].

Drucker, P. (1985). Innovation and Entrepreneuship, New York, Harper \& Row Publishers.

Elson, P., Hall, P. V. (2010). Strength, Size, Scope: A Survey of Social Enterprises in Alberta and British Columbia. Calgaryl Vancouver: Mount Royal University/ Simon Fraser University.

Encyclopédie d'Allembert et de Diderot. URL: http://portail.atilf.fr/cgi-bin/getobject_?a.37:216:2./var/artfla/encyclopedie/textdata /IMAGE [1 mai 2010].

Fontan, J.M. (2011). «Développement territorial et innovation sociale ». In Bellemare, G., Klein, J.L. Innovation sociale et territoire, convergences théoriques et pratiques. Québec, Presses de l'Université du Québec, Collection innovation sociale, 17-42.

Fontan, J.M. (1992). Les Corporations de développement économique communautaire montréalaises : du développement économique communautaire au développement local de l'économie. Montréal. Thèse de doctorat. Université de Montréal. Département de sociologie.

Fontan, J.M., Leblanc, J., Noiseux, Y., Silvestro, M. (2010). " Pratiques économiques solidaires québécoises en contexte de développement durable », dans Gendron, C., Vaillancourt, J.G., Audet, R. Développement durable et responsabilité sociale, de la mobilisation à l'institutionnalisation. Montréal. Presses internationales Polytechnique, 83-100.

Gould, S. (2006). « Social Enterprise and Business Structures in Canada ». Fraser Valley Centre for Social Enterprise. February.

Fulton, K., Dees, G. (2006). The Past, Present, and Future of Social Entrepreneurship. Centre for the Advancement of Social Entrepreneurship. New Profit Inc.

Hodgson, L. (2004). « Manufactured Civil Society: Counting the Cost». Critical Social Policy. Centre for Civil Society Studies, 24, 2(79), $139-164$.

Johnson, S. (2003). Young Social Entrepreneurs in Canada. Edmonton, AB: Canadian Centre for Social Entrepreneurship.

Johnson, S. (2000). Literature Review on Social Entrepreneurship. Edmonto, AB: Canadian Centre for Social Entrepreneurship.

Jetté, C. (2008). Les organismes communautaires et la transformation de l'État-Providence - Trois décennies de co-construction des politiques publiques dans le domaine de la santé et des services sociaux. Québec, Presses de l'Université du Québec.

Julien, P-A. (1994). «L'entreprise partagée: contraintes et avantages ». Gestion, 19(4), décembre, 48-58.

Keynes, J.M. ([1936], 1990). Théorie générale de l'emploi, de l'intérêt et de la monnaie. Paris. Bibliothèque scientifique Payot.

Klein, J.L., Fontan, J.M., Saucier, C., Tremblay, D.G., Tremblay, P.A., Simard, M. (2009). « Social Entrepreneurs, Local Initiatives and Social Economy: Foundations for a Socially Innovative Strategy to Fight against Poverty and Exclusion », Canadian Journal of Regional Science, XXXII(1), 23-42.

Klein, J.L., Harrisson, D. (dir.). (2007). L'innovation sociale. Émergence et effets sur la transformation des sociétés. Québec. Presses de l'Université du Québec.

Kramer, M.R. (2005). Measuring Innovation: Evaluation in the Field of Social Entrepreneurship. Palo Alto. The Skoll Foundation. Foundation Strategy Group.

Laville J.-L., Nyssens M. (2001). « The Social Enterprise: Towards a Theoretical Socio-Economic Approach ». In Borzaga C. \& Defourny J., (Eds.), The Emergence of Social Enterprise, London. Routledge, 312-332.

Leikin, S. (2005). Practical Utopians: American Workers and the Cooperative Movement in the Gilded Age. Detroit. Wayne State University Press. 


\section{Fontan (2011)}

Lévesque, B. (2002). Entrepreneurship collectif et économie sociale : entreprendre autrement. Montréal, Centre de rechreche sur les innovations sociales, cahier I-02-2002.

Lévy, A., Rival, M. (2010). « Des formes d'entreprenariat dans les associations, le cas des associations du secteur médico-social sous tutelle ». Politique et management public. 27(3). URL : http://halshs.archives-ouvertes.fr/docs/00/56/38/48/PDF/LA_vy_Rival_PMP 4.pdf [1 mai 2010].

MacPherson, I. (1979). Each for All: A History of the Co-operative Movement in English Canada, 1900-1945. Toronto. McClelland \& Stewart (Carleton Library Series).

Madill, J., Brouard, F., Hebb, T. (2009). « Conceptualizing Canadian Social Enterprises: Exploring Social Transformation, Financial Self-Sufficiency and Innovation ». 25th Annual CCSBE/CCPME 2009 Conference, Toronto, 16-18 octobre.

Manwaring, S., Valentine, A. (2011). « La situation de l'entreprise sociale au Canada ». Ottawa. Horizons, projet de recherche sur les politiques, février.

Marshall, A. (1898). Principles of Economics. An Introductory Volume. Londres. Macmillan.

Melville, H.D., Walesh, K. (1997). Grassroots Leaders for a New Economy: How Civic Entrepreneurs Are Building Prosperous Communities. San Francisco. Jossey-Bass Publishers.

Oxford English Dictionary online. (2003). URL : http://dictionary.oed.com [1 mai 2010].

Painter, A. (2006). « L'économie sociale au Canada - Concepts, données et mesure », Horizons, 8(2), 30-34.

Peredo, A.M., McLean, M. (2006). « Social Entrepreneurship: A Critical Review of the Concept ». Journal of World Business, 41, 56-65.

Petitclerc, M. (2007). Nous protégeons l'infortune. Les origines populaires de l'économie sociale au Québec. Montréal. VLB Éditeur.

Programme Leonardo da Vinci. (2002). Référentiel européen. Bruxelles. Séminaire d’Aix-en-Provence.

Restakis, J. (2006). Defining the Social Economy. The BC Context. Vancouver. BC Social Economy Roundtable. British Columbia Cooperative Association.

Riverin, N. (2006). L'entrepreneuriat social, une force tranquille. Chaire d'entrepreneuriat Rogers-J.A. Bombardier. HEC Montréal.

Robinson, J.A., \& Lo, J. (2005). Bibliography of Academic Papers on Social Entrepreneurship. Nyustern. Management and Organizations Departement.

Say, J.B. ([1803], 1972). Traité d'économie politique (1803) ou simple exposition de la manière dont se forment, se distribuent ou se consomment les richesses. Paris. Calmann-Lévy.

Salamon, L.M. (2002). « The Resilient Sector: the State of Nonprofit Sector in America ». In Salomom, L.M. (edit.). The State of Non Profit Sector in America, Washington (D.C.). Brooking Institution Press. Aspen Institute, XI-XVIII. URL : http://www.cof.org/files/Documents IEmerging_Issues/El\%20for\%20Philanthropy/Strategic\%20Relationships/chap10_-_Phil_Infrastructure___State_of_Nonprofit_America.pdf [1 août 2011].

Salmon, A. (2011). « L'entreprise providence. Espoir ou consternation? Débat sur la responsabilité sociale de l'entreprise (II) ». Revue du MAUSS permanente, mars. URL : http://www.journaldumauss.net/spip.php?article785 [1 août 2011].

Schumpeter, J.A. (1935). Théorie de l'évolution économique. Recherches sur le profit, le crédit, l'intérêt et le cycle de la conjoncture. Paris, Dalloz.

Smith, A., ([1776], 1904). An Inquiry into the Nature and Causes of the Wealth of Nations. London. Editor Edwin Cannan. Methuen and $C_{0}$. Fifth edition. 


\section{Fontan (2011)}

Spear, R. (2006). « Social entrepreneurship: a different model? ». International Journal of Social Economics, 33(5-6), 399-410.

Thompson, J., Alvy, G., Lees, A. (2000). Social Entrepreneurship: a new look at the people and the potential, Management Decision, MCB University Press.

Tounes, A. (2002). L'entrepreneur: l'odyssée d'un concept, IAE de Rouen. CREGO. Faculté de Droit, de Sciences Économiques et de Gestion, 03-73, 1-22.

Trivedi, C. (2011). «Social Enterprises and Corporate Enterprises: Fundamental Differences and Defining Features ». Journal of Entrepreneurship. 20, 1-32.

Trivedi, C. (2010a). « A social entrepreneurship bibliography ». Journal of Entrepreneurship, 19(1), 81-85.

Trivedi, C. (2010b). « Toward a social ecological framework for social entrepreneurship ». Journal of Entrepreneurship, 19(1), 63-80.

Tremblay, D.G., Klein, J.L., Fontan, J.M. (2009). Initiatives locales et développement socioterritorial. Montréal. Presses de l'Université du Québec et TÉLUQ.

Vanek, J. (1989). «De l'entreprise capitaliste à l'entreprise coopérative: problèmes et méthodes ». In Lévesque, B., Joyal, A., Chouinard, O. (Sous la dir.). L'autre économie : une économie alternative? Québec. Presses de l'Université du Québec, 235-244.

Van Ryzin, G.G.V., Grossman, S., DiPadova-Stocks, L., Bergrud, E. (2009). « Portrait of the social entrepreneur: Statistical evidence from a US Panel ». Voluntas, 20(2), 129-40.

Williams, S. (2005). Social Enterprise: The Three P's: Philosophy, Process and Practicalities. Edmonton. The Muttart Foundation.

Yalnizyan, A. (2007). Les Riches et nous : le visage changeant de l'écart croissant au Canada. Centre canadien de politiques alternatives. URL : http://www.growinggap.ca [1 août 2011].

\section{L'auteur / About the author}

Jean-Marc Fontan est professeur de sociologie, Université du Québec à Montréal. Courriel: fontan.jeanmarc@uqam.ca 